\title{
Patterns of initial failure after resection for gallbladder cancer: implications for adjuvant radiotherapy
}

\author{
Tae Gyu Kim, MD \\ Department of Radiation Oncology, Samsung Changwon Hospital, Sungkyunkwan University School of Medicine, \\ Changwon, Korea
}

Purpose: This study sought to identify potential candidates for adjuvant radiotherapy and patterns of regional failure in patients who underwent curative-intent surgery for gallbladder cancer.

Materials and Methods: Records for 70 patients with gallbladder cancer who underwent curative resection at a single institution between 2000 and 2016 were analysed retrospectively. No patients received adjuvant radiotherapy. Initial patterns of failure were evaluated. Regional recurrence was categorized according to the definitions of lymph node stations suggested by the Japanese Society of Hepato-Biliary-Pancreatic Surgery.

Results: Median follow-up was 23 months. Locoregional recurrence as any component of first failure occurred in 29 patients $(41.4 \%)$, with isolated locoregional recurrence in $13(18.6 \%)$. Regional recurrence occurred in 23 patients, and 77 regional recurrences were identified. Commonly involved regional stations were \#13, \#12a2, \#12p2, \#12b2, \#16a2, \#16b1, \#9, and \#8. Independent prognostic factors for locoregional recurrence were $\geq \mathrm{pT2}$ disease (hazard ratio [HR], 5.510; 95\% confidence interval [Cl], $1.260-24.094 ; p=0.023)$ and $\mathrm{R} 1$ resection $(H R, 6.981 ; 95 \% \mathrm{Cl}, 2.378-20.491 ; p<0.001)$.

Conclusion: Patients with pT2 disease or R1 resection after curative surgery for gallbladder cancer may benefit from adjuvant radiotherapy. Our findings on regional recurrence may help physicians construct a target volume for adjuvant radiotherapy.

Keywords: Gallbladder cancer, Adjuvant radiotherapy, Locoregional neoplasm recurrences, Lymph node, Lymphatic irradiation

\section{Introduction}

Gallbladder cancer is the most common malignancy of the biliary tract, and ranks sixth among gastrointestinal cancers [1]. Worldwide, there is a remarkable geographic variability in incidence of gallbladder cancer because it is related to the prevalence of cholelithiasis [2]. Korea has the highest incidence rate of gallbladder cancer in Asia: 8.1 per 100,000 for males and 5.6 for females [1]. Gallbladder cancer is one of the most aggressive tumors among gastrointestinal tract tumors and is associated with poor prognosis. Median survival is reported to be 44.0 months for patients with stage I cancer, 17.1 months for those with stage II, 9.0 months for stage III, and 3.4 months for stage IV $[3,4]$.

The most potentially curative therapy for gallbladder cancer is surgery. Because of the low probability of lymphatic metastasis in T1a gallbladder cancer, a simple cholecystectomy can provide a high cure rate [5]. Extended cholecystectomy is generally recommended for patients with gallbladder cancer at stage $\geq T 1 \mathrm{~b}$ because of the high probability of remnant

Received 11 August 2017, Revised 01 October 2017, Accepted 10 October 2017.

Correspondence: Tae Gyu Kim, MD, Department of Radiation Oncology, Samsung Changwon Hospital, Sungkyunkwan University School of Medicine, 158 Paryong-ro, Masanhoewon-gu, Changwon 51353, Korea. Tel: +82-55-233-6085, Fax: +82-55-233-5664, E-mail: tg1.kim@samsung.com

(c) This is an Open Access article distributed under the terms of the Creative Commons Attribution Non-Commercial License (http://creativecommons.org/ licenses/by-nc/4.0/) which permits unrestricted non-commercial use, distribution, and reproduction in any medium, provided the original work is properly cited.

www.e-roj.org 


\section{Tae Gyu Kim}

tumors and metastatic lymph nodes (LN) when performing only simple cholecystectomy [6,7]. Extended cholecystectomy includes cholecystectomy, en bloc hepatic resection, and lymphadenectomy with or without bile duct excision. Radical resection is the most proven method for improving the prognosis of gallbladder cancer patients [6]. However, the symptoms of patients with early gallbladder cancer are mild, and gallbladder cancer is often diagnosed at a progressive stage when surgery is difficult. In spite of attempts to improve resectability and survival, the prognosis of patients with gallbladder cancer remains poor due to high rates of local and distant metastasis after surgery [8].

Many studies have investigated whether adjuvant radiotherapy and/or chemotherapy lower recurrence rates and ultimately improve survival [9-16]. The effect of adjuvant treatment has not been clearly demonstrated due to the rarity of gallbladder cancer and the lack of randomized trials. Some, but not all, retrospective studies show a survival benefit with adjuvant radiotherapy or chemoradiotherapy. Benefits vary according to clinicopathological characteristics including tumour stage, extent of surgery, and resection margin status. When patients with a high probability of locoregional recurrence after surgery are selected, adjuvant radiotherapy may improve their oncologic outcomes.

To the best of our knowledge, the specific location of locoregional recurrence after surgery for gallbladder cancer has not been evaluated in order to establish radiation target volume. In the era of conformal radiotherapy such as threedimensional conformal radiotherapy (3D-CRT) or intensitymodulated radiation therapy (IMRT), knowledge of the location of locoregional recurrence could increase local control rates and minimize toxicity by improving radiation target identification.

The aim of this study was to determine prognostic factors for initial locoregional recurrence in gallbladder cancer patients who underwent curative-intent surgery without adjuvant radiotherapy. We also focused on identifying specific locations of regional recurrence to suggest possible radiotherapy target volumes.

\section{Materials and Methods}

The medical records of 133 consecutive patients who underwent surgical resection with curative intent for gallbladder cancer from January 2000 and December 2016 at Samsung Changwon Hospital were retrospectively reviewed. We excluded 8 patients who underwent R2 resection, 15 with
T2 or higher disease who did not undergo radical resection after a previous simple cholecystectomy, 17 who underwent postoperative radiotherapy, 10 without complete follow-up for recurrence patterns, 5 who died during the perioperative period, and 8 with a history of other malignancies. Records of remaining 70 patients were retrospectively analyzed. Informed consent for treatments was collected from all patients. This study was approved by the Institutional Review Board of Samsung Changwon Hospital (No. SCMC 2017-05-007).

The surgical approach was en bloc cholecystectomy and partial hepatectomy with regional lymphadenectomy with or without bile duct resection and reconstruction. Radical reoperation was performed for patients with T2 or higher disease who underwent a previous noncurative cholecystectomy. Patients who underwent previous simple cholecystectomy whose tumor was confined to the mucosa were typically not recommended for additional radical surgery. The extent of hepatic resection depended mainly on tumor location and the presence of major vascular involvement. Biliary resection and reconstruction was performed when tumors invaded the common hepatic or common bile duct.

Pathology reports were reviewed for prognostic factors including tumor stage, histological differentiation, presence of perineural invasion and lymphovascular invasion, resection margin status, LN involvement, and number of dissected LNs. The 7th edition of the American Joint Committee on Cancer criteria was used in staging.

The first postoperative follow-up evaluation was usually performed at 1 month after surgery. Patients were then followed every 3 months for the first 2 years and every 6 months for the subsequent 3 years. A complete history and physical examination, and tumor marker tests were performed at each visit. In general, computed tomography (CT) scans were performed every 6 months during the first 3 years and annually during the subsequent 2 years. Magnetic resonance imaging or positron emission tomography/computed tomography (PET/CT) were performed when recurrence was suspected from CT or tumor marker testing.

Patterns of recurrence were categorized as local (anastomotic site and tumor bed), regional (regional LN), or distant metastasis (other organs or non-regional LN metastasis). Locoregional recurrence was defined as any recurrence at locoregional area with or without distant metastasis, and isolated locoregional recurrence was defined as relapse at locoregional area without concomitant distant metastasis. The first site of recurrence during follow-up was evaluated by a single diagnostic radiologist. Location 
of regional recurrence was using definitions of LN stations suggested by the Japanese Society of Hepato-BiliaryPancreatic Surgery 3rd English edition [17]. Regional LN recurrence was diagnosed when (1) nodes reappeared after complete disappearance, or (2) new nodes appeared in regions where enlarged nodes had not existed before. When evaluating regional LNs using $\mathrm{CT}$ images, a short axis in the transverse plane greater than $1.0 \mathrm{~cm}$ was considered positive. Node location was defined as the position of the center point of the largest cross-section of the LN based on axial CT images (confluent LNs were counted as single node).

The locoregional recurrence-free survival and patterns of regional failure were defined as the study endpoints. Locoregional recurrence-free survival was plotted by Kaplan-Meier estimates. Univariable and multivariable Cox regression analyses were used to identify prognostic factors for recurrence and survivals. Patterns of regional recurrence were analyzed by computing the number and percentage of failure sites. All statistical analyses were performed using SPSS software ver. 20.0 (IBM, Armonk, NY, USA). A two-sided p-value $<0.05$ was considered statistically significant.

\section{Results}

\section{Patient characteristics}

Clinicopathological characteristics of patients are shown in Table 1. Median age was 67 years (range, 29 to 88 years). Approximately two-thirds of patients were female $(n=43$, 61.4\%). Pathologic T stage was T1 in 13 patients (18.6\%), T2 in $48(68.6 \%)$, and T3/4 in 9 (12.9\%); 22 patients (31.4\%) had pathologically positive LNs, and 9 (12.9\%) had $\mathrm{R} 1$ resection margins. The number of dissected LNs was $\geq 3$ in 35 patients (50.0\%) with a median value of 3 (range, 1 to 17 ). Median values for carcinoembryonic antigen (CEA) and carbohydrate antigen 19-9 (CA 19-9) at the time of diagnosis were 2.98 $\mathrm{ng} / \mathrm{mL}$ (range, 0.23 to $14.29 \mathrm{ng} / \mathrm{mL}$ ) and $13.7 \mathrm{U} / \mathrm{mL}$ (range, 0 to $6,430 \mathrm{U} / \mathrm{mL}$ ), respectively. Adjuvant chemotherapy with 5-fluorouracil, gemcitabine, cisplatin or capecitabine with single or combination regimen was administered to 19 patients $(27.1 \%)$.

\section{Survival}

Median follow-up was 23 months (range, 1 to 160 months). During the follow-up period, 38 patients (54.2\%) died. The 1 -year overall survival was $84.5 \%$ and 3 -year overall survival was $61.4 \%$. Recurrences were observed in 41 patients (58.5\%). The 1 -year disease-free survival was $72.5 \%$ and 3 -year
Table 1. Patients' characteristics

\begin{tabular}{|c|c|}
\hline Characteristic & Value \\
\hline Age (yr) & 67 (29-88) \\
\hline \multicolumn{2}{|l|}{ Sex } \\
\hline Male & 27 (38.6) \\
\hline Female & $43(61.4)$ \\
\hline \multicolumn{2}{|l|}{ Pathologic T stage } \\
\hline $\mathrm{T} 1 \mathrm{a}$ & $8(11.4)$ \\
\hline $\mathrm{T} 1 \mathrm{~b}$ & $5(7.1)$ \\
\hline T2 & $48(68.6)$ \\
\hline T3 & $8(11.4)$ \\
\hline T4 & $1(1.4)$ \\
\hline \multicolumn{2}{|l|}{ Pathologic N stage } \\
\hline No & $48(68.6)$ \\
\hline N1 & $17(24.3)$ \\
\hline N2 & $5(7.1)$ \\
\hline \multicolumn{2}{|l|}{ Histologic differentiation } \\
\hline Well differentiated & $41(58.6)$ \\
\hline Moderately differentiated & $14(20.0)$ \\
\hline Poorly differentiated & $9(12.9)$ \\
\hline Unspecified & $6(8.6)$ \\
\hline \multicolumn{2}{|l|}{ Perineural invasion } \\
\hline Yes & $10(14.3)$ \\
\hline No & $60(85.7)$ \\
\hline \multicolumn{2}{|l|}{ Lymphovascular invasion } \\
\hline Yes & $14(20.0)$ \\
\hline No & $56(80.0)$ \\
\hline \multicolumn{2}{|l|}{ Resection margin } \\
\hline Ro & $61(87.1)$ \\
\hline $\mathrm{R} 1$ & $9(12.9)$ \\
\hline \multicolumn{2}{|l|}{ No. of dissected lymph nodes } \\
\hline$<2$ & $35(50.0)$ \\
\hline$\geq 3$ & $35(50.0)$ \\
\hline CEA (ng/mL) & $2.98(0.23-50.23)$ \\
\hline CA19-9 (U/mL) & $13.7(0-6,430)$ \\
\hline \multicolumn{2}{|l|}{ Adjuvant chemotherapy } \\
\hline Yes & $19(27.1)$ \\
\hline No & 51 (72.9) \\
\hline
\end{tabular}

Values are presented as median (range) or number (\%).

CEA, carcinoembryonic antigen; CA19-9, carbohydrate antigen $19-9$.

disease-free survival was $51.2 \%$. The median time to initial recurrence was 9 months (range, 1 to 61 months). The period from initial recurrence to death was a median 6 months (range, 0 to 47 months) in relapsed patients.

\section{Patterns of failure}

Locoregional recurrence as first failure occurred in 29 patients (41.4\%), with isolated locoregional recurrence in 13 (18.6\%) 


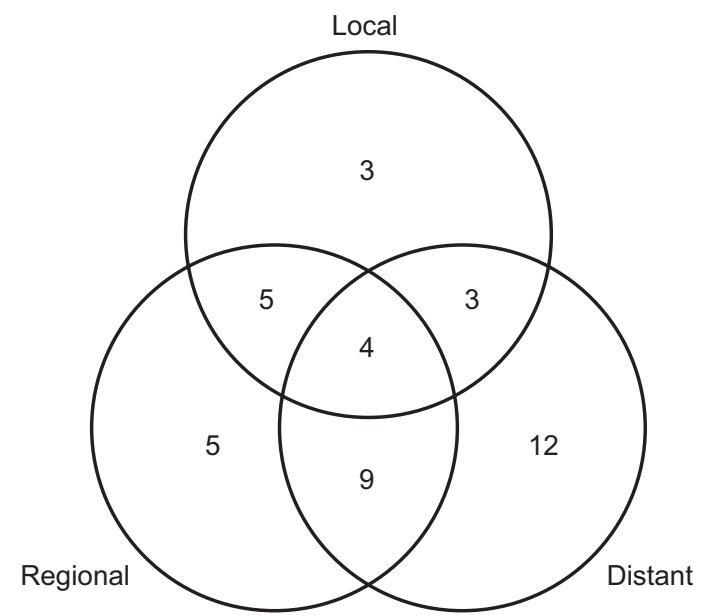

Fig. 1. Patterns of initial recurrence.

(Fig. 1). Distant metastasis with or without concomitant locoregional recurrence developed in 28 patients (40.0\%). Local recurrence occurred in 15 patients, of which 5 (33.3\%) failed at an anastomosis site, and 10 (66.7\%) in the tumor bed.

Regional recurrence occurred in 23 patients, and 77 regional recurrences were identified (Fig. 2). Of these, commonly involved stations were \#13 (LNs on the posterior surface of the head of the pancreas, 16.9\%), \#16a2 (LNs around the abdominal aorta between the upper border of the origin of the celiac artery and the lower border of the left renal vein, 14.3\%), and \#16b1 (LNs around the abdominal aorta between the lower border of the left renal vein and the upper border of the origin of the inferior mesenteric artery, 14.3\%). When \#8 (LNs along the common hepatic artery) and \#9 (LNs around the celiac artery), and \#12a2 (LNs along the proper hepatic artery in the caudal half between the confluence of the right and left hepatic ducts and the upper border of the pancreas), \#1262 (LNs on the anterior, posterior and right side surfaces of the bile duct in the caudal half between the confluence of the right and left hepatic ducts and the upper border of the pancreas), and \#12p2 (LNs on the posterior surface of the portal vein in the caudal half between the confluence of the right and left hepatic ducts and the upper border of the pancreas) categories were combined considering anatomical structure, recurrence at these sites were both 14.3\%. Recurrence at the \#7 (LNs along the trunk of left gastric artery), \#10 (LNs at the splenic hilum), \#11 (LNs along the splenic artery), \#12h (LNs in the hepatic side of the confluence of the right and left hepatic ducts), \#12a1 (LNs along the proper hepatic artery in the cranial half between the confluence of the right and left hepatic ducts and the upper border of the pancreas), \#12p1 (LNs on the posterior surface of the portal vein in the cranial half between the confluence of the right and left hepatic ducts and the upper border of the pancreas), \#12b1 (LNs on the anterior, posterior

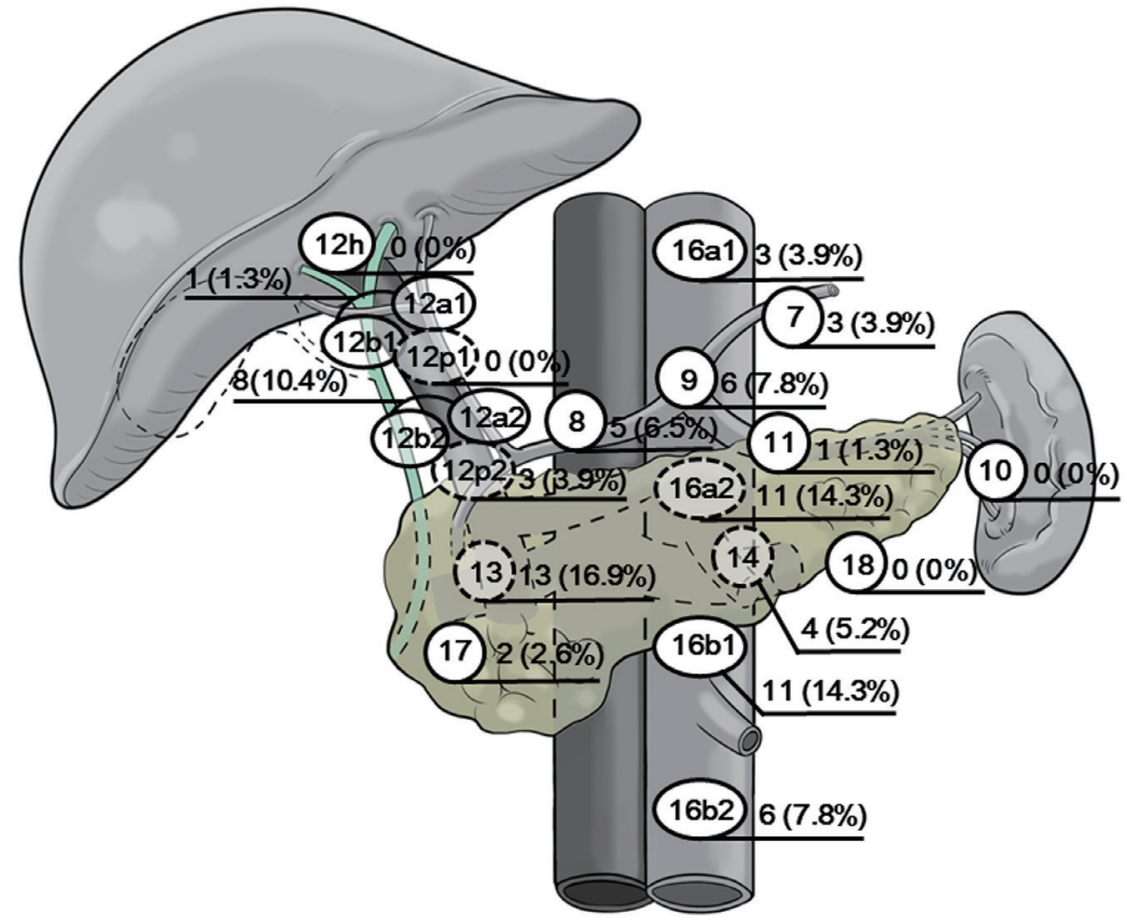

Fig. 2. Frequency of regional recurrence according to the criteria of the Japanese classification of biliary tract cancer. The number and frequency of the regional recurrences are indicated next to the lymph node station. 12a1 and 12b1, and 12a2 and $12 \mathrm{~b} 2$ nodes were counted together. 
Table 2. Prognostic factors of locoregional recurrence

\begin{tabular}{|c|c|c|c|c|}
\hline & \multicolumn{2}{|c|}{ Univariable } & \multicolumn{2}{|c|}{ Multivariable } \\
\hline & $\mathrm{HR}(95 \% \mathrm{CI})$ & $p$-value & $\operatorname{HR}(95 \% \mathrm{Cl})$ & p-value \\
\hline Age ( $\geq 70$ vs. $<70$ yr) & $1.156(0.553-2.419)$ & 0.699 & NA & \\
\hline Sex (male vs. female) & $1.152(0.799-1.661)$ & 0.448 & NA & \\
\hline Pathologic T stage ( $\geq$ T2 vs. T1) & $4.256(1.010-17.931)$ & 0.048 & $5.510(1.260-24.094)$ & 0.023 \\
\hline Pathologic N stage (N+ vs. N0) & $2.414(1.095-5.326)$ & 0.029 & $1.998(0.891-4.476)$ & 0.093 \\
\hline $\begin{array}{l}\text { Histologic differentiation (moderate-poorly } \\
\text { differentiated vs. well differentiated) }\end{array}$ & $1.997(0.894-4.461)$ & 0.092 & NA & \\
\hline Perineural invasion (yes vs. no) & $3.291(1.269-8.535)$ & 0.014 & $2.156(0.741-6.276)$ & 0.159 \\
\hline Lymphovascular invasion (yes vs. no) & $1.458(0.590-3.608)$ & 0.414 & NA & \\
\hline Resection margin (R1 vs. R0) & $4.926(1.888-12.852)$ & 0.001 & $6.981(2.378-20.491)$ & $<0.001$ \\
\hline No. of dissected lymph nodes (<2 vs. $\geq 3$ ) & $1.195(0.555-2.573)$ & 0.650 & NA & \\
\hline CEA ( $\geq 4$ vs. $<4 \mathrm{ng} / \mathrm{mL})$ & $1.499(0.514-4.372)$ & 0.459 & NA & \\
\hline CA19-9 ( $\geq 13$ vs. $<13 \mathrm{U} / \mathrm{mL})$ & $2.263(0.897-5.711)$ & 0.084 & NA & \\
\hline Adjuvant chemotherapy (no vs. yes) & $1.409(0.870-2.284)$ & 0.163 & NA & \\
\hline
\end{tabular}

$\mathrm{HR}$, hazard ratio; $\mathrm{Cl}$, confidence interval; NA, not applicable; CEA, carcinoembryonic antigen; CA19-9, carbohydrate antigen 19-9.
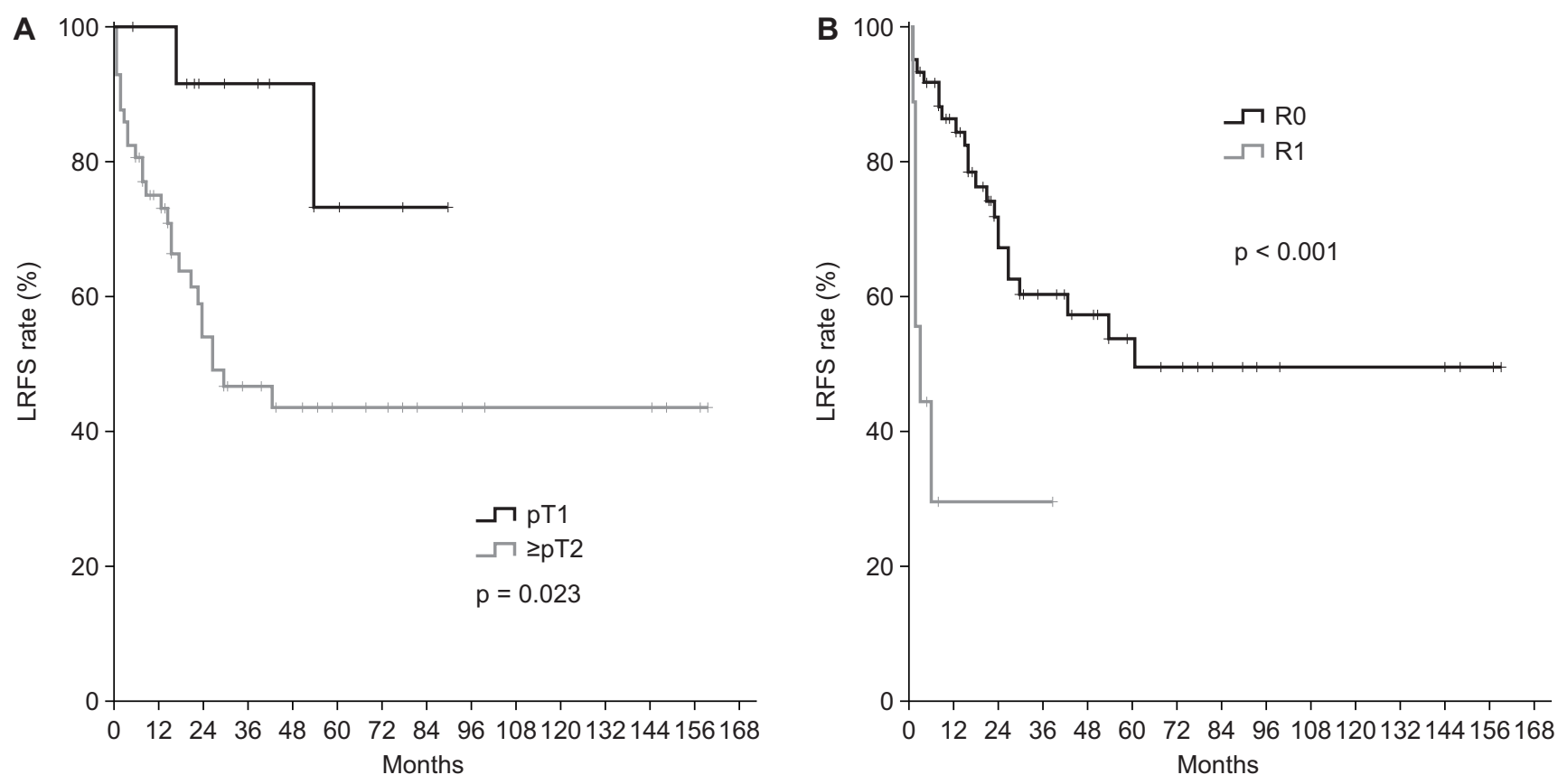

Fig. 3. Locoregional recurrence-free survival (LRFS) curves according to risk factors. (A) LRFS rates at 3 years were $91.7 \%$ in T1 patients and $46.1 \%$ in $\geq \mathrm{T} 2$ patients. (B) Three-year LRFS rates were $60.3 \%$ in patients with R0 resection, and $29.6 \%$ in patients with $\mathrm{R} 1$ resection.

and right side surfaces of the bile duct in the cranial half between the confluence of the right and left hepatic ducts and the upper border of the pancreas), \#15 (LNs along the middle colic vessels), \#16a1 (LNs around the abdominal aorta in the diaphragmatic aortic hiatus including infradiaphragmatic LNs predominantly along the subphrenic artery), \#17 (LNs on the anterior surface of the head of the pancreas), and \#18 (LNs along the inferior border of the pancreatic body and tail) stations was rare (0\%-3.9\%). Among 28 patients with distant metastasis, recurrence sites were peritoneum in 12 patients, liver in 10, lung in 5, and pancreas in 1.

\section{Factors related to recurrence and survivals}

Prognostic factors for locoregional recurrence as first failure were evaluated to identify potential adjuvant radiotherapy candidates (Table 2). Univariable analysis showed that pT stage, 
Table 3. Prognostic factors of overall survival

\begin{tabular}{|c|c|c|c|c|}
\hline & \multicolumn{2}{|c|}{ Univariable } & \multicolumn{2}{|c|}{ Multivariable } \\
\hline & $\mathrm{HR}(95 \% \mathrm{Cl})$ & p-value & $\mathrm{HR}(95 \% \mathrm{Cl})$ & p-value \\
\hline Age ( $\geq 70$ vs. <70 yr) & $1.267(0.626-2.564)$ & 0.511 & NA & \\
\hline Sex (male vs. female) & $1.001(0.699-1.433)$ & 0.996 & NA & \\
\hline Pathologic T stage ( $\geq T 2$ vs. T1) & $3.774(1.242-9.137)$ & 0.039 & $3.251(0.710-14.880)$ & 0.129 \\
\hline Pathologic N stage (N+ vs. N0) & $1.574(0.703-3.522)$ & 0.270 & & \\
\hline $\begin{array}{l}\text { Histologic differentiation (moderate-poorly } \\
\text { differentiated vs. well differentiated) }\end{array}$ & $2.058(0.984-4.307)$ & 0.055 & $2.180(0.975-4.874)$ & 0.058 \\
\hline Perineural invasion (yes vs. no) & $4.967(1.976-12.488)$ & 0.001 & $3.944(1.476-10.536)$ & 0.006 \\
\hline Lymphovascular invasion (yes vs. no) & $1.983(0.838-4.691)$ & 0.119 & NA & \\
\hline Resection margin (R1 vs. R0) & $5.973(2.167-16.460)$ & 0.001 & $10.125(2.937-34.903)$ & $<0.001$ \\
\hline No. of dissected lymph nodes (<2 vs. $\geq 3$ ) & $1.503(0.708-3.191)$ & 0.288 & NA & \\
\hline CEA ( $\geq 4$ vs. $<4 \mathrm{ng} / \mathrm{mL})$ & $1.764(0.660-4.715)$ & 0.258 & NA & \\
\hline CA19-9 ( $\geq 13$ vs. $<13 \mathrm{U} / \mathrm{mL})$ & $2.012(0.835-4.851)$ & 0.119 & NA & \\
\hline Adjuvant chemotherapy (no vs. yes) & $1.153(0.770-1.724)$ & 0.490 & NA & \\
\hline
\end{tabular}

$\mathrm{HR}$, hazard ratio; $\mathrm{Cl}$, confidence interval; NA, not applicable; CEA, carcinoembryonic antigen; CA19-9, carbohydrate antigen 19-9.

$\mathrm{pN}$ stage, presence of perineural invasion, and $\mathrm{R} 1$ resection were significantly associated with locoregional recurrence. In multivariable analysis, independent prognostic factors for locoregional recurrence were $\geq \mathrm{T} 2$ disease (hazard ratio $[\mathrm{HR}], 5.510 ; 95 \%$ confidence interval [Cl], 1.260-24.094; $\mathrm{p}=$ $0.023)$ and $\mathrm{R} 1$ resection $(\mathrm{HR}, 6.981 ; 95 \% \mathrm{Cl}, 2.378-20.491 ; p$ $<0.001$ ) (Fig. 3). Pathological positive node was of borderline significance ( $p=0.093)$.

Prognostic factors for overall survival are shown in Table 3. Presence of perineural invasion, $\mathrm{pT}$ stage, and $\mathrm{R} 1$ resection were significant factors of overall survival in univariable analysis. Among these factors, presence of perineural invasion ( $\mathrm{HR}, 3.944 ; 95 \% \mathrm{Cl}, 1.476-10.536 ; p=0.006)$ and $\mathrm{R} 1$ resection ( $H R, 10.125 ; 95 \% \mathrm{Cl}, 2.937-34.903 ; p<0.001)$ were independent prognostic factors for overall survival.

Fig. 4 demonstrates the effect of locoregional recurrence on overall survival. The 1 -year overall survival was $80.5 \%$ in patients with locoregional recurrence and $87.2 \%$ in patients without locoregional recurrence. The survival rate difference between the two groups rapidly increased with time.

\section{Discussion and Conclusion}

Although survival has increased with the development of surgical techniques for gallbladder cancer, overall prognosis is still poor due to a high rate of locoregional and distant metastasis. It has been reported that in contrast to patients with margin-positive resections, in whom locoregional recurrences predominate, the pattern of disease recurrence

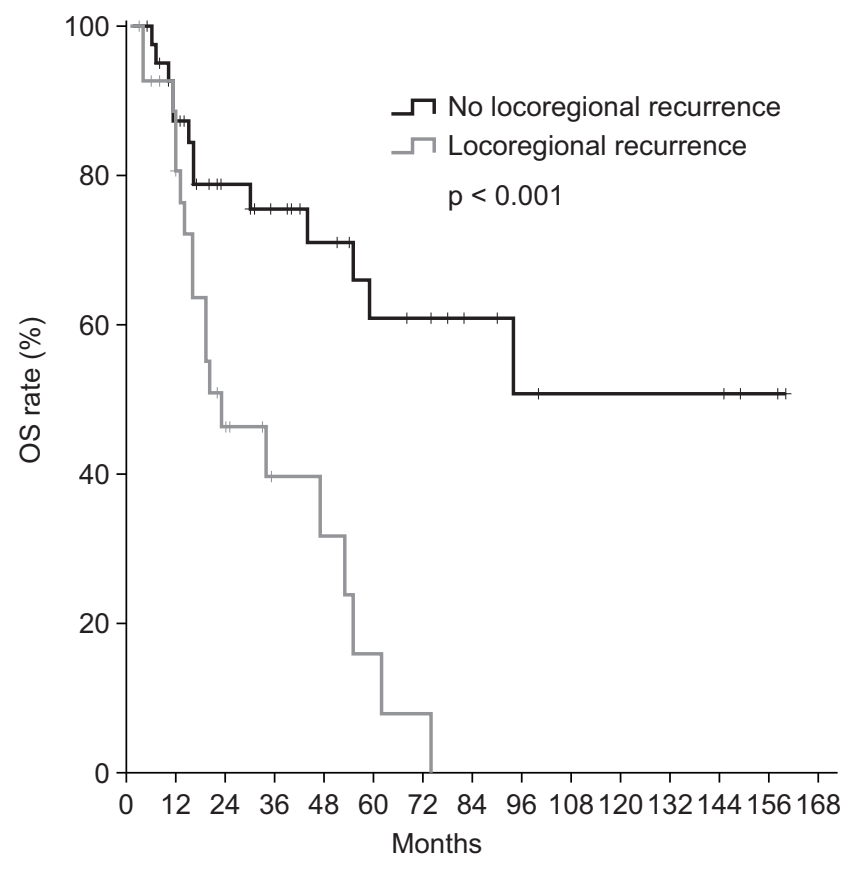

Fig. 4. Overall survival (OS) curves according to locoregional recurrence. The 3 -year overall survival was $39.6 \%$ in patients with locoregional recurrence and $75.4 \%$ in patients without locoregional recurrence.

following complete resection is predominantly distant. Jarnagin et al. [18] showed that local recurrence occurred in 13 patients $(17 \%)$, regional recurrence in $8(11 \%)$, and distant metastasis in $55(72 \%)$ in a study of 80 patients who underwent potentially curative resection for gallbladder cancer. Of the patients who developed recurrence, only $15 \%$ had isolated locoregional 
disease without concomitant distant metastasis. Jung et al. [19] evaluated initial patterns of failure of 61 gallbladder cancer patients who underwent curative-intent surgery, and showed that locoregional recurrence occurred in 19 (31.1\%). Isolated locoregional recurrence occurred in 7 (29.2\%) of 24 patients with recurrence as any component of first failure. Kim et al. [20] reported that 53 of 166 patients (31.9\%) who received curative-intent surgery for gallbladder cancer developed tumor recurrence. Among 53 patients with relapse, 8 (15.1\%) had isolated locoregional recurrence, and distant metastasis with or without concomitant locoregional recurrence occurred in 21 (39.6\%). The authors of those studies suggested that the usefulness of adjuvant radiotherapy is limited because distant metastasis is more prevalent than locoregional recurrence after radical surgery for gallbladder cancer. However, the studies included some patients who received adjuvant radiotherapy, which may be a limitation in assessing accurate locoregional recurrence patterns.

In studies of patients who received adjuvant radiotherapy or chemoradiotherapy for gallbladder cancer, locoregional recurrence rates were even lower. The Southwest Oncology Group conducted a phase II trial (SWOG S0809) which was designed to evaluate the role of adjuvant chemoradiotherapy in extrahepatic cholangiocarcinoma or gallbladder cancer [21]. In 25 gallbladder cancer patients, concurrent local and distant recurrence occurred in 2 patients (8\%), distant-only recurrence in 11 patients (44\%), and no one developed isolated local recurrence. Jeong et al. [22] evaluated 86 patients with gallbladder cancer who underwent postoperative radiotherapy, and found that first site of failure was isolated locoregional recurrence in 7 patients (8\%), distant metastases in 36 (42\%), and both in 6 (7\%). In this study evaluating patients who underwent curative-intent surgery for gallbladder cancer without adjuvant radiotherapy, isolated locoregional recurrence occurred in 13 patients (18.6\%) and concurrent locoregional and distant recurrence in 29 (41.4\%). Compared with previous studies in patients who received adjuvant radiotherapy, many patients included in this study may have been able to reduce locoregional recurrence through adjuvant radiotherapy.

Use of adjuvant radiotherapy with or without concomitant chemotherapy for treatment of gallbladder cancer is poorly established. Park et al. [11] evaluated the effect of adjuvant therapy on survival after curative resection in 61 patients with stage II gallbladder cancer, and found no survival benefit from adjuvant therapy. Glazer et al. [12] showed that adjuvant therapy did not significantly prolong survival after resection with wide negative margins in a study of 63 gallbladder cancer patients. In contrast, other studies reported that adjuvant radiotherapy is beneficial under certain conditions. Mojica et al. [23] conducted a Surveillance, Epidemiological, and End Results (SEER) database analysis of 3,187 patients, and showed that median survival for patients who received adjuvant radiotherapy was 14 months compared to 8 months for patients treated without adjuvant radiotherapy ( $p \leq 0.001)$. The benefits of adjuvant radiotherapy appeared mainly to be for patients with regional spread $(p<0.0001)$ and tumors infiltrating the liver $(p=0.011)$. In another analysis using SEER database of 4,000 patients performed by Wang et al. [15], adjuvant radiotherapy was a significant predictor of overall survival, and adjuvant radiotherapy was most beneficial for patients with LN involvement or $\geq T 2$ disease. Mitin et al. [24] evaluated 5,029 patients diagnosed with T1-3N0-1 gallbladder cancer using the National Cancer Database (NCDB), and demonstrated that adjuvant radiotherapy was associated with improved three-year overall survival (HR, 0.63; 95\% $\mathrm{Cl}, 0.44-0.92)$. Another study using NCDB of 4,775 patients showed that $\mathrm{T} 3$ or node-positive disease treated with adjuvant chemoradiotherapy had a modest early survival advantage (absolute difference at 2 years $=6.8 \%, p=0.009$ ) in inverse probability of treatment weighting analysis to minimize treatment related bias [25]. In this study, $\geq T 2$ disease and $\mathrm{R} 1$ resection were independent prognostic factors for locoregional recurrence, and locoregional recurrence was independent prognostic factor for overall survival. Therefore, patients with $\geq T 2$ disease and $\mathrm{R} 1$ resection are expected to benefit from local control through adjuvant radiotherapy.

Locoregional recurrence with or without concomitant distant metastasis was evaluated to find out potential candidates for adjuvant radiotherapy in this study. Finding prognostic factors of isolated locoregional recurrence might be more appropriate for the purposes of this research. Although not presented in the results section, $\geq T 2$ disease and $\mathrm{R} 1$ resection were significant prognostic factors of isolated locoregional recurrence in multivariable analysis ( $H R$, 5.336; 95\% Cl, 1.226-23.230; $p=0.026 ; \mathrm{HR}, 5.499 ; 95 \% \mathrm{Cl}$, 1.816-16.656; $p=0.003$, respectively). However, even when both distant and locoregional recurrence are detected at the same time, locoregional recurrence is likely to occur first. And patients with distant metastasis may have better oncologic outcomes through the development of systemic chemotherapy. Therefore, locoregional recurrence was presented as endpoint in this study.

With the development of radiotherapy techniques, 
3D-CRT or IMRT has become widely used to treat abdominal malignancies, allowing optimal qualitative treatment with reduced toxicity [26]. In addition, Sun et al. [27] conducted plan comparison between conformal radiotherapy and IMRT in the treatment of gallbladder cancer, and showed that IMRT reduced radiation dose to liver and right kidney, significantly. Defining accurate and precise target volume is important for planning these advanced techniques. Traditional radiation target volume is established by considering the primary nodal drainage of the gallbladder, which is to LNs along the cystic and common bile ducts, then to pancreaticoduodenal nodes and to para-aortic nodes. Marinelli et al. [28] conducted a systematic review of three pathology studies (247 patients) to suggest recommendations for target volume delineation for patients with unresectable gallbladder cancer. The review suggested the LN stations to include in target volume due to high rate of metastasis are: pericholedocal (frequency = $25.2 \%)$, cystic duct (23.0\%), retroportal (17.1\%), common hepatic artery (15.8\%), hepatoduodenal ligament (13.4\%), retropancreatic (12.3\%), pancreatic duodenal anterior and posterior (7.7\%), para-aortic (6.0\%), and hilar LN (5.1\%). However, no research has established a target volume design for adjuvant radiotherapy by revealing detailed LN stations of regional recurrences in gallbladder cancer. In our study evaluating initial recurrence patterns in patients who underwent curative-intent surgery for gallbladder cancer, commonly involved nodal regions included LNs of the caudal half-hepatoduodenal ligament, on the posterior surface of the pancreatic head, along the common hepatic artery, around the celiac artery, and around the abdominal aorta between the origin of the celiac artery and the origin of the inferior mesenteric artery. We suggest that these nodal regions and gallbladder fossa should be included in target volumes of adjuvant radiotherapy, assuming that the target volume includes regions with a recurrence frequency of $10 \%$ or more.

Results of this retrospective study should be interpreted with caution. First, a median of three LNs were dissected in patients who underwent radical surgery, which is a relatively small number compared with other studies. The small number of resected LNs was thought to contribute to the relatively high locoregional recurrence rate. Also, patients who underwent $\mathrm{R} 2$ resection and/or patients with $\geq T 2$ disease who did no undergo radical resection after a previous simple cholecystectomy were not included in this study. Therefore, the results of this study may be difficult to apply when more extended lymphadenectomy or insufficient surgery are performed. Second, recognizing and determining recurrence in a small or infiltrative lesion by clinical radiographic detection is difficult. This challenge could indicate limitations in the assessment of actual recurrence patterns. The strength of this study is that we studied patients who did not undergo adjuvant radiotherapy for identifying accurate locoregional recurrence patterns after surgery.

In conclusion, despite curative-intent surgery, gallbladder cancer patients, especially those with $\geq \mathrm{T} 2$ disease or $\mathrm{R} 1$ resection margin were at substantial risk of locoregional recurrence. The considerable risk of locoregional failure inducing poor quality of life and survival in these patients justifies adding adjuvant radiotherapy to control for failures. Our findings on the prevalence of regional nodal failure could provide important information to physicians for constructing clinical target volumes for adjuvant radiotherapy.

\section{Conflict of Interest}

No potential conflict of interest relevant to this article was reported.

\section{References}

1. Hundal R, Shaffer EA. Gallbladder cancer: epidemiology and outcome. Clin Epidemiol 2014;6:99-109.

2. Randi G, Franceschi S, La Vecchia C. Gallbladder cancer worldwide: geographical distribution and risk factors. Int $J$ Cancer 2006;118:1591-602.

3. Miller G, Jarnagin WR. Gallbladder carcinoma. Eur J Surg Oncol 2008;34:306-12.

4. Fong $Y$, Wagman $L$, Gonen $M$, et al. Evidence-based gallbladder cancer staging: changing cancer staging by analysis of data from the National Cancer Database. Ann Surg 2006;243:76774.

5. Lee SE, Kim KS, Kim WB, et al. Practical guidelines for the surgical treatment of gallbladder cancer. J Korean Med Sci 2014;29:1333-40.

6. Sikora SS, Singh RK. Surgical strategies in patients with gallbladder cancer: nihilism to optimism. J Surg Oncol 2006;93:670-81.

7. Abramson MA, Pandharipande P, Ruan D, Gold JS, Whang EE. Radical resection for $T 1 b$ gallbladder cancer: a decision analysis. HPB (Oxford) 2009;11:656-63.

8. Margonis GA, Gani F, Buettner $S$, et al. Rates and patterns of recurrence after curative intent resection for gallbladder cancer: a multi-institution analysis from the US Extra-hepatic Biliary Malignancy Consortium. HPB (Oxford) 2016;18:872-8. 
9. Mallick S, Benson R, Haresh KP, Julka PK, Rath GK. Adjuvant radiotherapy in the treatment of gall bladder carcinoma: what is the current evidence. J Egypt Natl Canc Inst 2016;28:1-6.

10. Itoh H, Nishijima K, Kurosaka $Y$, et al. Magnitude of combination therapy of radical resection and external beam radiotherapy for patients with carcinomas of the extrahepatic bile duct and gallbladder. Dig Dis Sci 2005;50:2231-42.

11. Park HS, Lim JY, Yoon DS, et al. Outcome of adjuvant therapy for gallbladder cancer. Oncology 2010;79:168-73.

12. Glazer ES, Liu P, Abdalla EK, Vauthey JN, Curley SA. Neither neoadjuvant nor adjuvant therapy increases survival after biliary tract cancer resection with wide negative margins. J Gastrointest Surg 2012;16:1666-71.

13. Williams TM, Majithia L, Wang SJ, Thomas CR Jr. Defining the role of adjuvant therapy: cholangiocarcinoma and gall bladder cancer. Semin Radiat Oncol 2014;24:94-104.

14. Gold DG, Miller RC, Haddock MG, Gunderson LL, Quevedo F, Donohue JH, Bhatia S, Nagorney DM. Adjuvant therapy for gallbladder carcinoma: the Mayo Clinic Experience. Int J Radiat Oncol Biol Phys 2009;75:150-5.

15. Wang SJ, Fuller CD, Kim JS, Sittig DF, Thomas CR Jr, Ravdin PM. Prediction model for estimating the survival benefit of adjuvant radiotherapy for gallbladder cancer. J Clin Oncol 2008;26:2112-7.

16. Kim MY, Kim JH, Kim Y, Byun SJ. Postoperative radiotherapy appeared to improve the disease free survival rate of patients with extrahepatic bile duct cancer at high risk of loco-regional recurrence. Radiat Oncol J 2016;34:297-304.

17. Miyazaki M, Ohtsuka M, Miyakawa S, et al. Classification of biliary tract cancers established by the Japanese Society of Hepato-Biliary-Pancreatic Surgery: 3(rd) English edition. J Hepatobiliary Pancreat Sci 2015;22:181-96.

18. Jarnagin WR, Ruo $L$, Little $S A$, et al. Patterns of initial disease recurrence after resection of gallbladder carcinoma and hilar cholangiocarcinoma: implications for adjuvant therapeutic strategies. Cancer 2003;98:1689-700.
19. Jung SJ, Woo SM, Park HK, et al. Patterns of initial disease recurrence after resection of biliary tract cancer. Oncology 2012;83:83-90.

20. Kim WS, Choi DW, You DD, Ho CY, Heo JS, Choi SH. Risk factors influencing recurrence, patterns of recurrence, and the efficacy of adjuvant therapy after radical resection for gallbladder carcinoma. J Gastrointest Surg 2010;14:679-87.

21. Ben-Josef E, Guthrie KA, El-Khoueiry AB, et al. SWOG S0809: a phase II intergroup trial of adjuvant capecitabine and gemcitabine followed by radiotherapy and concurrent capecitabine in extrahepatic cholangiocarcinoma and gallbladder carcinoma. J Clin Oncol 2015;33:2617-22.

22. Jeong $Y$, Park JH, Lee $Y$ J, et al. Postoperative radiotherapy for gallbladder cancer. Anticancer Res 2014;34:5621-9.

23. Mojica $P$, Smith D, Ellenhorn J. Adjuvant radiation therapy is associated with improved survival for gallbladder carcinoma with regional metastatic disease. J Surg Oncol 2007;96:8-13.

24. Mitin $T$, Enestvedt $C K$, Jemal $A$, Sineshaw HM. Limited use of adjuvant therapy in patients with resected gallbladder cancer despite a strong association with survival. J Natl Cancer Inst 2017;109:djw324.

25. Mantripragada KC, Hamid F, Shafqat H, Olszewski AJ. Adjuvant therapy for resected gallbladder cancer: analysis of the National Cancer Data Base. J Natl Cancer Inst 2016;109:djw202.

26. Bae JS, Kim DH, Kim WT, Kim YH, Park D, Ki YK. The role of surgical clips in the evaluation of interfractional uncertainty for treatment of hepatobiliary and pancreatic cancer with postoperative radiotherapy. Radiat Oncol J 2017;35:65-70.

27. Sun XN, Wang $0, G u B X$, et al. Adjuvant radiotherapy for gallbladder cancer: a dosimetric comparison of conformal radiotherapy and intensity-modulated radiotherapy. World J Gastroenterol 2011;17:397-402.

28. Marinelli I, Guido A, Fuccio L, et al. Clinical target volume in biliary carcinoma: a systematic review of pathological studies. Anticancer Res 2017;37:955-61. 